\title{
Metabonomics research accelerates discovery of medical biomarkers
}

\author{
Yanning Cai ${ }^{1}$, Qian Dong ${ }^{1}$ \\ ${ }^{1}$ College of information Science and Technology, Jinan University, Guangzhou, China
}

\begin{abstract}
Biomarker refers to a characteristic that can be objectively detected and evaluated, and can be used as an indicator of normal biological process, pathological process or therapeutic intervention pharmacological response. As one of the key words of individualized medicine, the search and discovery of valuable biomarkers has become a research hotspot in the current medical field.
\end{abstract}

\section{Introduction}

The first tumor marker can be traced back to the Bence Jones protein confirmed by H. Bence Jones in 1846, which has certain diagnostic value for multiple myeloma[1]. More than 170 years later, with the platform of genomics, proteomics, Peptidomics and metabonomics, as well as the means and methods including nanotechnology, bioinformatics, antibody chip and other cutting-edge technologies, it is possible to obtain and screen biomarkers quickly[2][3].

Genomics and transcriptomics describe the physiological indicators that may change, while metabolites are the end products of signaling pathways, reflecting the physiological state of the body at that time. Compared with other omics, metabonomics is more suitable for the study of disease classification and marker discovery because it is closer to phenotype[4][5].

In 2009, researchers from Howard Hughes School of medicine, University of Michigan, found biomarker sarcosine of prostate cancer based on GC-MS metabonomics research platform, and found that it may play an important role in cancer cell metastasis [6]. In 2011, scientists used LC-MS metabonomics research platform to find biomarkerof cardiovascular disease, some phospholipid metabolites, such as choline, trimethylamine oxide and betaine[7]. In 2014, the domestic research team used the GC-MS metabonomics research platform to find the diagnosis biomarker of acute myeloid leukemia, and found another set of metabolic mechanism of tumor cells through further research, and revealed the possible therapeutic target [8]. In 2014, the US team used LC-MS metabonomics research platform to find four biomarkers to distinguish different stages of lung cancer development, and conducted population validation of more than 1000 cases [9]. In 2016, the first batch of large sample metabonomics research in China used LC-MS metabonomics research platform to screen 12 groups of biomarker with high sensitivity and strong specificity, which can be used for rapid diagnosis of clinical coronary heart disease and differential diagnosis of different types [10]. In 2017, a metabolic dynamic image called "metabonomics" has been used to identify new blood metabolite biomarkers that can distinguish pancreatic cancer from chronic pancreatitis [11][12]. Compared with traditional diagnostic methods, it has higher sensitivity and is suitable for the early stage of disease. The researchers reported that biomarker characteristics distinguished pancreatic cancer from chronic pancreatitis, assuming cumulative incidence rate of $1.95 \%, 0.96$ under the curve, $89.9 \%$ sensitivity and $91.3 \%$ specificity. This has successfully verified the characteristics of biomarkers.

\section{The research route of biomarker discovery in metabonomics}

Metabonomics through the high-throughput detection of body metabolites, combined with multivariate statistical analysis, screening significant differences in metabolic markers, and then from the overall deep dialysis of the pathological mechanism of the disease, providing a scientific basis for the prevention and treatment of diseases [13]. Fig.1 shows the relationship between metabonomics and biomarkers in diagnosis and treatment. The research route is as Fig.2.

caiyanningmitl@sina.com 

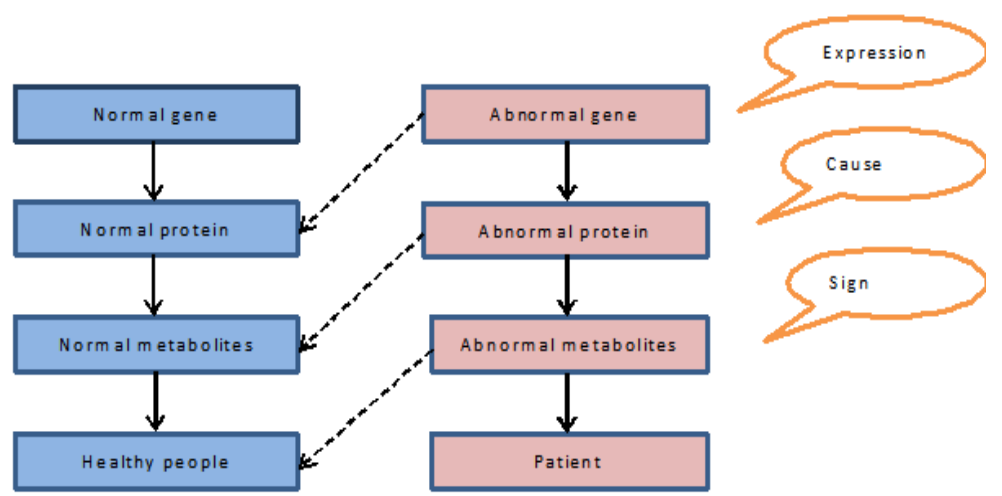

Figure 1. Metabonomics and biomarkers $n$.

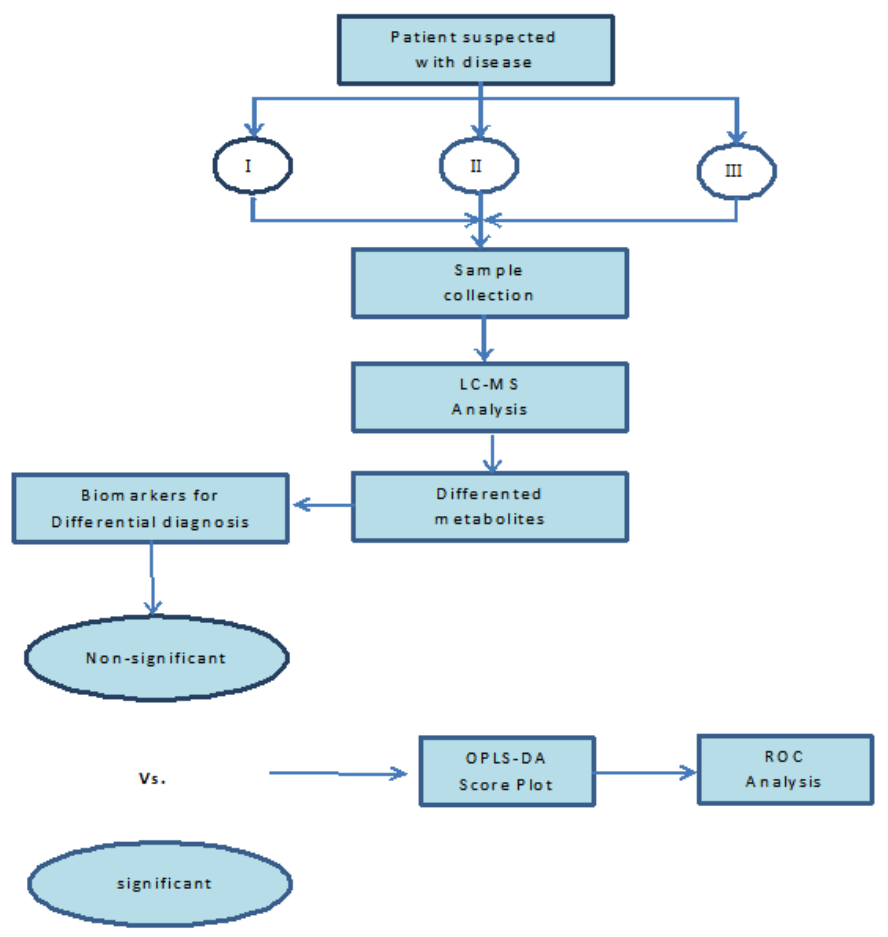

Figure 2. Metabonomics discovery biomarker research route

\section{Application of metabonomics in disease classification and biomarker discovery}

The metabolism of life is a never-ending process. The metabolism of any substance in the body will significantly affect the development of body fluids [14][15]. A group of biomarkers related to the diagnosis and classification of diseases can be found by comparing the body physiology and disease status, metabolite differences of different types and stages of the same disease [16][17].

As a kind of cardiovascular disease, coronary heart disease is a common disease that seriously endangers people's life and health [18]. Although the current general digital subtraction angiography has a high diagnosis rate, it is traumatic, expensive, with many side effects and cumbersome operation. Early diagnosis of coronary heart disease with small trauma, low cost and easy operation is urgently needed [19].

Metabonomics can be used to detect small molecular substances with high throughput through standard biological samples such as serum, plasma and urine. Many of these small molecules have been considered as risk factors of diseases [20].

Researchers from Cleveland hospital and UCLA found that the "by-products" produced by intestinal bacteria degrading lecithin can predict who may have heart disease in the future. Further animal experiments have shown that intestinal microbiota plays an important role in the conversion of dietary lipids into trimethylamine $\mathrm{N}$-oxide (TMAO), which promotes the formation of arterial plaques leading to cardiovascular disease [21]. These studies suggest that intestinal symbiotic bacteria can also cause or aggravate diseases 
including obesity and immune abnormalities, and drugs targeting TMAO may prevent atherosclerosis and heart disease.

After finding the key biomarker, how can we further study the disease mechanism? In the era of omics, with the development of genomics, proteomics, Peptidomics, metabonomics and so on, as well as the development of bioinformatics, it has become possible to explain and elaborate the pathological mechanism of diseases by multi omics joint analysis [22]. In 2016, based on previous metabonomics research and combined with genomics, researchers of the team conducted mGWAS (metabolome genome wide association study). They took plasma from 3880 patients with coronary heart disease, and used metabonomic technology to detect choline and other (8 kinds of) metabolites, and obtained SNP information of materials by re sequencing [23]. Based on metabonomic data and re sequencing SNP information, mgwas research was carried out. It was concluded that two loci on chromosome $2 \mathrm{q} 34$ and $5 \mathrm{q} 14.1$ were significantly related to the development of coronary artery disease. Glycine metabolism and urea cycle may reveal a new mechanism of atherosclerotic disease development.

In addition to cancer and cardiovascular diseases, in recent years, with the improvement of living standards, high-fat, high protein diet, lack of exercise, and some unhealthy living habits, lead to the "metabolic syndrome" composed of high-risk factors such as obesity, hypertension, hyperglycemia, which threaten the health of modern people. The use of metabonomics technology can also be used for the pathogenesis and diagnosis of these diseases And drug efficacy provides new clues [24].

\section{Expectation: searching for the advantages of biomarker by targeting metabonomics extensively}

Widely targeted metabolomics is a new technology for qualitative and quantitative detection of metabolites. It combines the advantages of non targeting and targeting, and integrates the advantages of high throughput, high sensitivity and quantification. It is especially suitable for the detection of low and medium abundance metabolites. It is the most suitable method for the discovery of new metabolic biomarker, with high throughput (detection of more than 1000 metabolites at a time) and high sensitivity

( It can detect low abundance metabolites, wide coverage (covering more than 20 metabolic pathways), and accurate qualitative and quantitative analysis (with nearly 1000 kinds of standard sample library). The metabolites detected include amino acids and their derivatives, sugars, nucleotides and their derivatives, vitamins, lipids and other substances [25].

Through gene sequencing to find the disease control genes, through protein sequencing to study how genes regulate downstream signaling pathways, and finally through metabonomics analysis to study the final results of gene expression regulation. The combined analysis of genomics, transcriptome, proteome and metabonomics can help us to understand the pathogenesis of diseases and find new entry points for diagnosis, monitoring and treatment of diseases.

\section{Acknowledgment}

This work was supported by the Natural Science Foundation of Guangdong Province (Grants No. 2018030310074), Youth Fund Project of Jinan University research and Innovation Fund (Grants No. 21617350).

\section{References}

1. Abraham R S, Clark R J , Bryant S C, et al. Correlation of Serum Immunoglobulin Free Light Chain Quantification with Urinary Bence Jones Protein in Light Chain Myeloma[J]. Clinical Chemistry, 2002, 48(4):655-7.DOI: 10.1016/S00098981(01)00766-5.

2. JinongL, Zhen $Z$, Jason $R$, et al. Proteomics and Bioinformatics Approaches for Identification of Serum Biomarkers to Detect Breast Cancer[J]. Clinical Chemistry(8):1296. DOI: 10.1016/S00098981(02)00170-5.

3. Cheng L ,Meiser B . The relationship between psychosocial factors and biomarkers in cancer patients: A systematic review of the literature[J]. European Journal of Oncology Nursing, 2019, 41. DOI: $10.1007 / \mathrm{s} 11136-018-2045-7$.

4. Jiang $\mathrm{H}$, Liu $\mathrm{J}$, Qin $\mathrm{X} \mathrm{J}$, et al. Gas chromatography-time of flight/mass spectrometry-based metabonomics of changes in the urinary metabolic profile in osteoarthritic rats[J]. Experimental \& Therapeutic Medicine, 2018, 15(3):2777-2785. DOI: $10.3892 /$ etm.2018.5788.

5. Li H D ,Xu Q S , Liang Y Z . libPLS: An Integrated Library for Partial Least Squares Regression and Discriminant Analysis[J]. Chemometrics and Intelligent Laboratory Systems, 2018, 176:34-43. DOI: 10.7287/peerj.preprints.190v1.

6. SreekumarA, Poisson L M, Rajendiran T M, et al. Metabolomic profiles delineate potential role for sarcosine in prostate cancer progression[J]. Nature, 2009, 457(7231):910-914. DOI: 10.1038 /nature07762.

7. Wang Z ,Klipfell E, Bennett B J , et al. Gut flora metabolism of phosphatidylcholine promotes cardiovascular disease[J]. Nature, 2011, 472(7341):57-63. DOI: 10.1038/nature07762.

8. Wenming Chen, Peng Wei, Shifang Yang, et al. Transiently Elevated AST/LDH Are Associated with Clinical Response to Recombinant Circularly Permuted TRAIL (CPT) Plus Thalidomide in Patients with Relapsed and/or Refractory Multiple Myeloma[J]. Blood, 2014, 124:3478-3478. DOI: 10.1085/jgp.49.6.103.

9. Lu M , Jolly M K, Onuchic J, et al. Toward Decoding the Principles of Cancer Metastasis Circuits[J]. Cancer Research, 2014, 74(17):45744587. DOI: 10.1158/0008-5472.CAN-13-3367. 
10. Dong D, Huihui Z, Xueli L, et al. GW27-e0313 Investigation of correlation between Gensini score and TCM syndrome with unstable angina in coronary heart disease[J]. Journal of the American College of Cardiology, 2016, 68(16):C91. DOI: 10.1016/j.jacc.2016.07.341.

11. Mayerle J, Kalthoff H, Reszka R, et al. Metabolic biomarker signature to differentiate pancreatic ductal adenocarcinoma from chronic pancreatitis[J]. Gut, 2018, 67(1):128-137. DOI: 10.1136/gutjnl-2016312432.

12. Costello, Eithne. A metabolomics-based biomarker signature discriminates pancreatic cancer from chronic pancreatitis[J]. Gut, 2018:gutjnl-2016313665. DOI: 10.1136/gutjnl-2016-313665.

13. Lindon J C, Holmes E, Bollard M E, et al. Metabonomics technologies and their applications in physiological monitoring, drug safety assessment and disease diagnosis[J]. Biomarkers, 2010, 9(1):131. DOI: $10.1080 / 13547500410001668379$.

14. SathasivamR, Radhakrishnan R, Hashem A, et al. Microalgae metabolites: A rich source for food and medicine[J]. Saudi Journal of Biological ences, 2019, 26:709-722. DOI: 10.1016/j.sjbs.2017.11.003.

15. Hart G W . Nutrient Regulation of Signaling \&Transcription[J]. Journal of Biological Chemistry, 2019, 294(7):jbc.AW119.003226. DOI: 10.1074/jbc.AW119.003226.

16. JinongL, Zhen $Z$, Jason $R$, et al. Proteomics and Bioinformatics Approaches for Identification of Serum Biomarkers to Detect Breast Cancer[J]. Clinical Chemistry, 2020, (8):1296. DOI: 10.1016/S0009-8981(02)00170-5.

17. O'Neill H J , Gordon S M, O'Neill M H , et al. A computerized classification technique for screening for the presence of breath biomarkers in lung cancer.[J]. Clinical Chemistry, 2019, (8):1613. DOI: 10.1016/S0009-9120(88)80010-9.

18. Li M, Wang X, Li X, et al. Statins for the Primary Prevention of Coronary Heart Disease[J]. BioMed Research International, 2019, 2019:1-15. DOI: $10.1155 / 2019 / 4870350$.

19. Hsue P Y, Waters D D . HIV infection and coronary heart disease: mechanisms and management[J]. Nature Reviews Cardiology, 2019. DOI: 10.1038/s41569-019-0219-9.

20. Kempa E E, Hollywood K A, Smith C A, et al. High throughput screening of complex biological samples with mass spectrometry - from bulk measurements to single cell analysis[J]. Analyst, 2019.

21. Wang Z ,Klipfell E, Bennett B J , et al. Gut flora metabolism of phosphatidylcholine promotes cardiovascular disease[J]. Nature, 2011, 472(7341):57-63. DOI: 10.1039/c8an01448e.

22. XinjunW, Zhe $S$, Yanfu $Z$, et al. BREM-SC: a bayesian random effects mixture model for joint clustering single cell multi-omics data[J]. Nucleic Acids Research(11):11. DOI: 10.1093/nar/gkaa314.
23. Lusis A J ,Seldin M, Allayee H, et al. The Hybrid Mouse Diversity Panel: a resource for systems genetics analyses of metabolic and cardiovascular traits[J]. Journal of Lipid Research, 2016:925-942. DOI: 10.1194/jlr.R066944.

24. Armindo Miguel de Jesus Sousa de AraújoRibeiro. Metabolic Syndrome[M]// Metabolic Disorders [Working Title]. 2019.

25. A G C, B Z S , D Y H A, et al. Large-scale Targeted Metabolomics Method for Metabolite Profiling of Human Samples[J]. 